\title{
Editorial
}

\section{What place for oral surgery during the epidemics of SARS-Cov2?}

\author{
Géraldine Lescaille* \\ Université de Paris, Department of odontology of Pitié-salpêtrière Hospital of Paris, Paris, France
}

(Received: 26 April 2020, accepted: 27 April 2020)

Since the end of December 2019, date of the identification of the first cluster in China, SARS-CoV2 has quickly spread to become pandemic with a global mortality rate estimated between 2 and 3\%, which could go up to $12 \%$ in the northern Italy. Most forms of this infection are asymptomatic or mild, very commonly associated with anosmia and ageusia, but can also cause severe pneumonia. Among the identified patients, $15 \%$ of the confirmed cases of Covid-19 have developed severe forms, including very few pediatric cases. The mortality rate in intensive care is around 25\% in France, especially in elderly patients with associated comorbidities, but can also affect young patients with no medical history. On the other hand, if Covid-19 was initially considered as an exclusively respiratory disease, it now appears that it is also a systemic disease. Although of multifactorial origin (high age, immobilization, obesity, active cancer, drugs, etc.) the thromboembolic risk is very high in the cases of Covid-19, and is particularly associated with a marked inflammatory syndrome. Another hypothesis relating to a direct toxicity of the virus on the vascular endothelium could explain the observed vasculitis, the skin lesions or to better understand the origin of the anosmia potentially related to the necrosis of small nerve terminals, or even the destruction of the endothelium of the small vessels of the lung. Potential odontologic manifestations, including mucosal ulcers related to Covid-19 are under investigation.

At present, although the combination of hydroxychloroquine and azithromcyine may be useful, no treatment has been verified as scientifically proven. Several other clinical trials are underway. While the situation seems to be back to normal in China, cases are increasing worldwide and seem to be stabilizing in several European countries, in particular thanks to mass confinement, but this plateau could last several months. To fight the epidemic in France, dental offices and universities closed their doors on Monday, March 16, and all training, seminars, symposia have been postponed, including our annual congress of the SFCO scheduled for May 2020 has been postponed to May 2021. During stage 3 of Covid19 epidemic, only oral emergencies are maintained, with specific measures to avoid cross-contamination, and organized by the departmental councils of the order of dentists, or in dental emergencies department of hospital. Personal protective equipment (PPE) are reinforced to take into account the risks associated with aerosolization due to the use of high speed dental instruments, and is associated with a strict organization that provides for ventilation of rooms and space planning measures. Several studies are underway to identify in particular the risk of cross-contamination, in particular with aerosolization. As such, an epidemiological survey on the involvement of practicing practitioners was initiated with the implementation of longitudinal monitoring. These preventive measures must be respected over time with the scheduled deconfinement by selecting the acts that can be performed in optimal conditions after May 11, recently announced by French government. Dental learned societies have recently done theirs pespectives published by the National Authority for Health.

\footnotetext{
* Correspondence: geraldine.lescaille@gmail.com
} 